\title{
On the relation between information structure and predictive inference in text understanding
}

\author{
SIMON BORCHMANN
}

This article presents a pragma-linguistic refinement of the focus model (Sanford \& Garrod 1981). The aim of the refinement is to determine the relation between information structure and predictive inferences. The refinement is the result of theoretical work made in preparation to a series of experiments testing a number of specific hypotheses about this relation. The article is restricted to the results of the preliminary theoretical work. In the article it is argued that previous research in text driven inferences has been based on insufficient or even misleading accounts of natural language information structure, and thus, on an unclear correlation of the linguistic and the psychological concept of focus. As a consequence the role of new focus in sentence integration is left unexplained. It is suggested that this problem can be solved by co-ordinating psychological accounts of text understanding on the one hand, and pragma-linguistic accounts of natural language (Togeby 1993 \& 2003, Heltoft 2003, Borchmann 2005) and schema theory (Winograd 1977, Schank 1999, Minsky 1985), on the other. On this basis the following hypothesis is proposed: Under certain conditions, the information structure constituent new focus occasions an expectation, namely if the focus represents an insufficient level of specification ("level-band" (cf. Minsky 1985)) in proportion to the schema relevant to the solution of the problem dealt with by the text. The involvement of new focus can explain previous experimental evidence, and the specification of the condition is in line with a number of pragmatic observations. Thus, involving pragma-linguistic accounts of natural language in psychological research in text driven inferences is warranted. 